
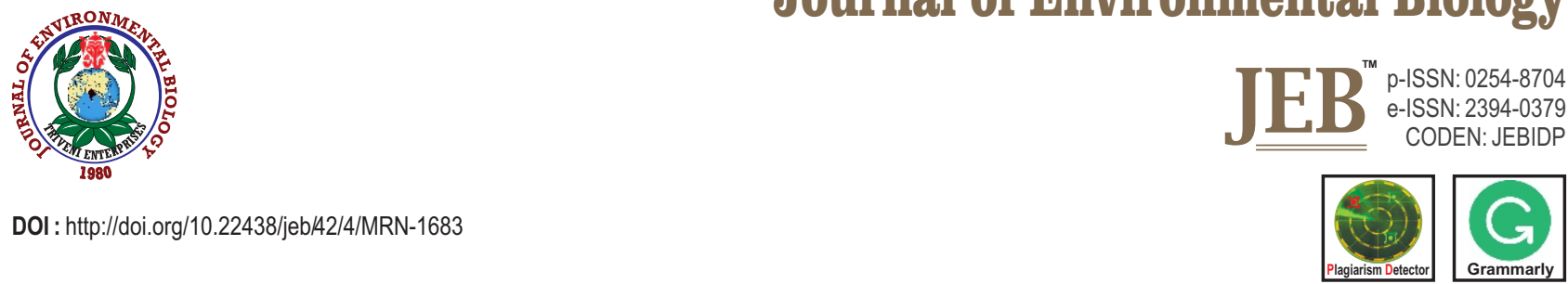

\title{
Response of cotton genotypes against the incidence of Alternaria leaf blight of cotton under field conditions
}

\author{
G. Rajesha ${ }^{1 *}$, S. Nakkeeran ${ }^{2}$, T. Indumathi' ${ }^{2}$ P. Adhipathi ${ }^{2}$ and A. Chandrasekar ${ }^{2}$ \\ 'Department of Plant Pathology, ICAR-Indian Institute of Millets Research, Hyderabad-500 030, India \\ ${ }^{2}$ Department of Plant Pathology, Tamil Nadu Agricultural University, Coimbatore-641 003, India \\ *Corresponding Author Email : rajeshag337@gmail.com
}

Received: 28.08 .2020

Revised: 07.11.2020

Accepted: 20.02 .2021

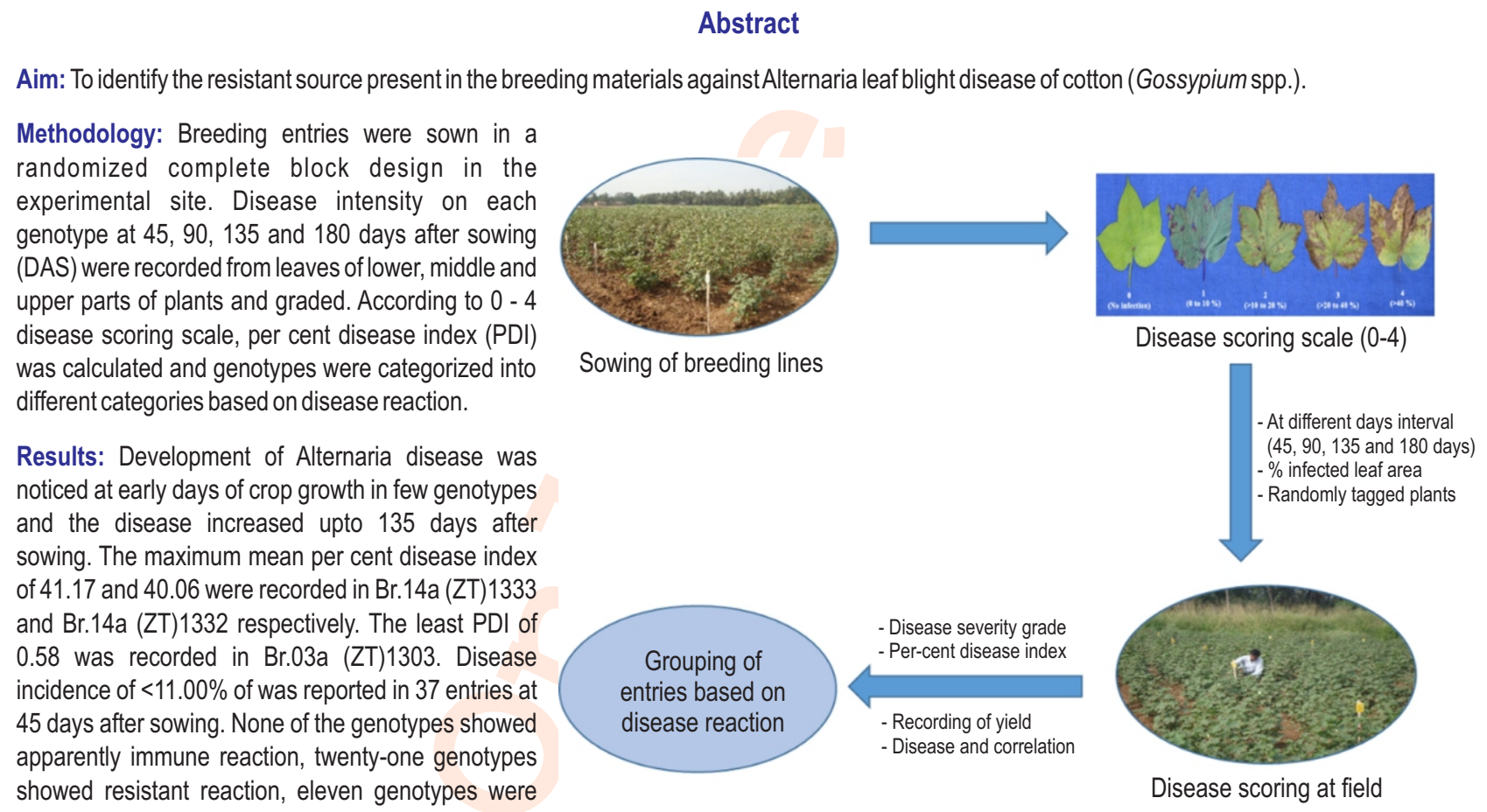

moderately resistant and five showed moderately susceptible reaction.

Interpretation: Twenty-one breeding lines showed resistant reaction against Alternaria leaf blight disease under field conditions, however, further utilization of these lines may be carried out in breeding programmes for the development of resistant source against this disease.

Key words: Alternaria leaf blight, Cotton, Disease reaction, Genotypes

How to cite : Rajesha, G., S. Nakkeeran, T. Indumathi, P. Adhipathi and A. Chandrasekar: Response of cotton genotypes against the incidence of Alternaria leaf blight of cotton under field conditions. J. Environ. Biol., 42, 1002-1007 (2021). 


\section{Introduction}

Cotton (Gossypium spp.) known as the "king of fibers" is an important fiber and cash crop occupying nearly 33 million ha in 77 countries as a major source of natural fiber worldwide (Singh and Rathore, 2020). It is widely grown in India and is one of the important livelihood for an estimated 60 million Indian people, including 6 million farmers, mostly small and marginal. In addition to fiber production, it is also used for gossypol production due to its extensive range of biological properties including anti-cancer, antimicrobial, anti-HIV, anti-oxidative and male contraceptive activities (Vander et al., 2000). It is being cultivated as a major crop in parts of the African Tropics, Australia, China, Egypt, India, New Mexico, Pakistan, Soviet Union, Sudan, United States and warmer regions of Central and South America (Leff et al., 2004). India is the second world producer of cotton after China, but account for only $18 \%$ of total world cotton production against $23 \%$ of world cotton area. Cotton crop is continually exposed to threat of biotic stress and considered as a major biological challenge to exploit cotton productivity.About $80-90 \%$ of disease in cotton pertains to foliar diseases (Gulhane and Gurjar, 2011).

Among them, Alternaria leaf blight is one of the major foliar disease, prevalent in all cotton growing regions worldwide including Israel, India, China, Egypt, Russia, Zimbabwe, New South Wales, Australia and the United States (Newman, 2011). Average disease severity index ranged from 21.5 to $87.0 \%$ was reported in New Mexico (Zhu et al., 2019). In India, this disease is one of the economic foliar disease of cotton (Bhattiprolu and Rao, 2013), which appears every year and has reduced the cotton production (Cui et al., 2000). The yield loss of 37 per cent was reported in India by Padaganur et al. (1989) due to occurrence of this pathogen. In Tamil Nadu, the incidence of leaf blight disease increased to 54.62 PDI during the year 2013-2014 and caused severe yield loss of $32 \%$ (Saravanan et al., 2015). The leaf blight symptom occurs due to two species of Alternaria ( $A$. macrospora Zimm. and A. alternata (Fr.) Keissler) and are common in cotton crops grown around the world (Kaur and Aggarwal, 2015). The species A. macrospora causes sudden premature defoliation resulting in severe yield loss whereas $A$. alternat a infection is also prominent on cotton, but is usually less damaging (Hillocks, 1991; Rajesha et al., 2020).

Several disease outbreaks have occurred mainly in cotton growing regions in the world and caused yield losses upto $25 \%$ in Israel (Rotem et al., 1988). The yield reduction of $38.23 \%$ (Bashi et al., 1983; Bhattiprolu and Rao, 2009) was reported due to increased incidence and severity, resulting in severe defoliation in the late cotton growing season (Zhu et al., 2018), especially when it affects highly susceptible cultivars. Resistant crops provide an effective and inexpensive method for managing diseases in both high value as well as low value cropping systems. They are environmentally compatible, safe to use and do not require specialized applications, as faced in chemical application (Fitt et al., 2002). The use of resistant cultivar has proved to be most desirable method of Alternaria leaf blight management. Although no commercial cotton cultivars grown are known to be immune to Alternaria, and no breeding program is known to develop Alternaria resistant cotton cultivars, different levels of Alternaria leaf blight resistance may exist in current commercial cotton cultivars and breeding lines (Zhu et al., 2018). Bodhke et al. (2019) evaluated thirteen varieties against Alternaria leaf blight of cotton, only two varieties were found resistant reaction and remaining were susceptible to disease. Significant variation in disease severity index (DSI) ranged from $26.7 \%$ to $92 \%$ was recorded among the 160 genotypes of cotton in New Mexico (Zhu et al., 2018). Thus, it is important to evaluate the disease reaction on available cultivars. In view of the above, this study was conducted to evaluate resistance source among genotypes of cotton for Alternaria leaf blight disease.

\section{Materials and Methods}

Experimental set up: A total of thirty-nine breeding entries were sown in a randomized complete block design for evaluation under field conditions at Department of Cotton, Tamil Nadu Agricultural University, Coimbatore, India. Land was prepared well and brought to good tilth condition suitable for sowing. The field was sown by breeding entries with plot size of $10 \times 5 \mathrm{~m}\left(50 \mathrm{~m}^{2}\right)$ with 3 replications. The local recommended package of practices of cotton cultivation, Tamil Nadu were practiced without using any pesticides and fungicides application during experimentation.

Field evaluation of cotton genotypes against Alternaria leaf blight of cotton: Evaluation of cotton breeding entries against Alternaria leaf blight disease was carried out to identify the source of resistance under field conditions. Thirty-nine breeding entries were screened by recording observations on the intensity of Alternaria leaf blight disease on each genotype at 45, 90, 135 and 180 days after sowing (DAS). Total of five leaves from lower, middle and upper parts from ten plants in each genotype were randomly selected and percent leaf area was calculated according to 0 - 4 disease scoring scale of following criteria: 0 - No infection, completely free from disease; 1 - Few $<2 \mathrm{~mm}$, scattered brown spots and 0.1 to $10 \%$ infected leaf area covered; 2 - Spots bigger, $3 \mathrm{~mm}$, not coalescing, brown and $11-20 \%$ infected leaf area; 3 - Spots 3-5 mm, irregular in shape-coalescing, 21-40\% infected leaf area; 4 - Spots coalescing to form bigger lesions, irregular $>40$ infected leaf area (Sheo Raj, 1988). Based on the PDI, genotypes were categorized into immune, highly resistant, moderately resistant, moderately susceptible and highly susceptible based on the disease reaction (Sheo Raj, 1988). In addition to disease incidence, an average seed cotton yield of all the selected 10 plants of each genotypes were recorded and expressed in gram per plant.

Statistical analysis: The data was analyzed independently. The treatment means were compared by Duncan's Multiple RangeTest (DMRT) (Gomez and Gomez, 1984). The package used for analysis was IRRISTAT version 92-1 developed by the International Rice Research Institute Biometrics unit, the Philippines. 


\section{Results and Discussion}

Identification of disease resistant source is essential to use as donors for the resistant source in breeding programmes and recommendation for cultivation in high disease prone areas. The plants of durable disease resistance are effective to cultivate in larger area with out yield loss (Consortum, 2016). The resistance identified cultivars consistently resulted in less disease than non-resistant cultivator in high pathogen population
(Wheeler et al., 1999). Field evaluation is a useful and convenient method for identifying cotton genotypes with natural resistance to Alternaria leaf blight (Zhu et al., 2018). In the present study, breeding materials were screened under field conditions and percent disease index (PDI) was recorded at 45, 90, 135 and 180 days after sowing (Table 1). The results revealed that development of disease was noticed at early days of crop growth in few genotypes and the increased PDI was observed upto 135 days after sowing (DAS) during flowering and boll

Table 1: Percent disease index (PDI) and seed cotton yield/plant due to Alternaria leaf blight after different days of sowing of cotton germplasm under field conditions

\begin{tabular}{|c|c|c|c|c|c|c|}
\hline \multirow{2}{*}{ Name of entries } & \multicolumn{4}{|c|}{ Per cent Disease Index (PDI)* } & \multirow{2}{*}{$\begin{array}{l}\text { Mean } \\
\text { PDI }\end{array}$} & \multirow{2}{*}{$\begin{array}{l}\text { Seed cotton } \\
\text { yield/plant }(\mathbf{g})^{*}\end{array}$} \\
\hline & 45 DAS & 90 DAS & 135 DAS & 180 DAS & & \\
\hline Br.03a (ZT)1301 & 0.00 & 2.32 & 3.75 & 2.25 & 2.08 & $184.00^{c-9}$ \\
\hline Br.03a (ZT)1302 & 0.00 & 0.00 & 3.75 & 2.00 & 1.44 & $186.00^{\text {c.f }}$ \\
\hline Br.03a (ZT)1303 & 0.00 & 3.00 & 4.57 & 5.00 & 3.14 & $191.00^{\text {bee }}$ \\
\hline Br.03a (ZT)1304 & 6.7 & 7.75 & 17.95 & 9.2 & 10.4 & $179.33^{\mathrm{c-i}}$ \\
\hline Br.03a (ZT)1305 & 6.75 & 10.50 & 16.25 & 7.50 & 10.25 & $171.67^{f-1}$ \\
\hline Br.03a (ZT)1306 & 0.00 & 5.12 & 3.75 & 3.75 & 3.16 & $184.33^{c-g}$ \\
\hline Br.03a (ZT)1307 & 0.00 & 0.00 & 1.12 & 1.59 & 0.68 & $206.00^{\mathrm{ab}}$ \\
\hline Br.03a (ZT)1308 & 0.00 & 2.32 & 0.00 & 0.00 & 0.58 & $208.67^{a}$ \\
\hline Br.03a (ZT)1309 & 0.00 & 0.00 & 2.00 & 2.76 & 1.19 & $195.33^{\mathrm{a}-\mathrm{d}}$ \\
\hline Br.04a (ZT)1311 & 3.75 & 11.43 & 17.50 & 9.79 & 10.62 & $170.67^{f-m}$ \\
\hline Br.04a (ZT)1312 & 5.15 & 15.25 & 12.25 & 7.50 & 10.04 & $164.67^{\text {h-o }}$ \\
\hline Br.04a (ZT)1313 & 3.76 & 8.72 & 3.75 & 5.25 & 5.37 & $179.33^{\text {ci. }}$ \\
\hline Br.04a (ZT)1314 & 3.93 & 3.75 & 12.55 & 7.43 & 6.92 & $185.67^{\text {c-f }}$ \\
\hline Br.04a (ZT)1315 & 0.00 & 8.30 & 3.75 & 3.75 & 3.95 & $181.67^{\mathrm{chh}}$ \\
\hline Br.04a (ZT)1316 & 3.50 & 4.00 & 0.00 & 0.00 & 1.88 & $193.33^{\mathrm{a}-\mathrm{e}}$ \\
\hline Br.04a (ZT)1317 & 3.75 & 8.50 & 3.75 & 5.70 & 5.43 & $170.67^{f-m}$ \\
\hline Br.04a (ZT)1318 & 0.00 & 4.20 & 5.20 & 3.00 & 3.10 & $195.67^{\mathrm{abc}}$ \\
\hline Br.05a (ZT)1321 & 0.00 & 3.25 & 3.75 & 5.75 & 3.19 & $178.33^{\mathrm{dj} j}$ \\
\hline Br.05a (ZT)1322 & 0.00 & 9.75 & 12.85 & 20.20 & 10.70 & $168.33^{\mathrm{g}-\mathrm{n}}$ \\
\hline Br.05a (ZT)1323 & 0.00 & 11.55 & 3.75 & 8.31 & 5.90 & $171.33^{f-1}$ \\
\hline Br.05a (ZT)1324 & 0.00 & 0.00 & 3.50 & 3.00 & 1.63 & $195.33^{a-d}$ \\
\hline Br.05a (ZT)1325 & 5.75 & 8.31 & 16.56 & 10.32 & 10.24 & $176.33^{\mathrm{e}-\mathrm{k}}$ \\
\hline Br.05a (ZT)1326 & 0.00 & 5.00 & 5.00 & 9.12 & 4.78 & $186.67^{c-f}$ \\
\hline Br.14a (ZT)1331 & 11.25 & 27.57 & 28.75 & 18.50 & 21.52 & $159.67^{k-q}$ \\
\hline Br.14a (ZT)1332 & 32.72 & 38.50 & 46.25 & 42.75 & 40.06 & $145.67^{\mathrm{pq}}$ \\
\hline Br.14a (ZT)1333 & 37.28 & 41.72 & 47.55 & 38.12 & 41.17 & $143.67^{9}$ \\
\hline Br.14a (ZT)1334 & 5.25 & 15.32 & 18.75 & 15.75 & 13.77 & $161.67^{j-p}$ \\
\hline Br.14a (ZT)1335 & 8.37 & 16.70 & 21.75 & 18.30 & 16.28 & $158.33^{1-q}$ \\
\hline Br.14a (ZT)1336 & 7.50 & 18.89 & 15.00 & 12.00 & 13.35 & $166.33^{h-0}$ \\
\hline Br.14a (ZT)1337 & 9.08 & 22.75 & 27.20 & 24.05 & 20.77 & $159.67^{k-q}$ \\
\hline Br.14a (ZT)1338 & 9.00 & 18.65 & 11.25 & 14.50 & 13.35 & $163.67^{i-0}$ \\
\hline Br.14a (ZT)1339 & 9.75 & 24.75 & 29.75 & 19.85 & 21.03 & $153.33^{n-q}$ \\
\hline Br.15a (ZT)1351 & 6.75 & 11.65 & 15.25 & 8.54 & 10.55 & $162.00^{-p}$ \\
\hline Br.15a (ZT)1352 & 0.00 & 5.55 & 3.75 & 2.75 & 3.01 & $170.00^{t-n}$ \\
\hline Br.15a (ZT)1353 & 0.00 & 0.00 & 5.90 & 4.30 & 2.55 & $181.67^{c-h}$ \\
\hline Br.15a (ZT)1354 & 0.00 & 0.00 & 4.20 & 5.70 & 2.48 & $194.67^{a-d}$ \\
\hline Br.15a (ZT)1355 & 11.00 & 28.90 & 37.50 & 35.67 & 28.27 & $149.67^{\text {opq }}$ \\
\hline Br.15a (ZT)1356 & 7.90 & 23.25 & 28.75 & 24.55 & 21.11 & $153.67^{\mathrm{m}-\mathrm{q}}$ \\
\hline Br.15a (ZT)1357 & 0.00 & 0.00 & 2.70 & 4.60 & 1.83 & $189.33^{\text {cde }}$ \\
\hline
\end{tabular}

*Values are the mean of three replications 
Table 2: Disease reaction, percent leaf area covered and number of entries of cotton germplasms againstAlternaria leaf blight under field condition

\begin{tabular}{|c|c|c|c|c|}
\hline $\begin{array}{l}\text { Disease Severity } \\
\text { Grade }\end{array}$ & $\begin{array}{l}\text { Disease } \\
\text { reaction }\end{array}$ & $\begin{array}{l}\text { Per cent leaf } \\
\text { area covered }\end{array}$ & $\begin{array}{l}\text { Number of } \\
\text { entries }\end{array}$ & Entries \\
\hline 0 & Immune & 0.00 & - & $\begin{array}{l}- \\
\text { Br.03a (ZT)1301, Br.03a (ZT)1302, Br.03a } \\
\text { (ZT)1303, Br.03a (ZT)1306, Br.03a (ZT)1307, } \\
\text { Br.03a (ZT)1308, Br.03a (ZT)1309, Br.04a } \\
\text { (ZT)1313, Br.04a (ZT)1314, Br.04a (ZT)1317, }\end{array}$ \\
\hline 1 & Highly Resistant & Up to 10 & 21 & $\begin{array}{l}\text { Br.04a (ZT)1316, Br.04a (ZT)1318, Br.05a } \\
\text { (ZT)1321, Br.05a (ZT)1323, Br.05a (ZT)1324, } \\
\text { Br.05a (ZT)1326, Br.15a (ZT)1351, Br.15a } \\
\text { (ZT)1352, Br.15a (ZT)1353, Br.15a (ZT)1354, } \\
\text { Br.15a (ZT)1357 } \\
\text { Br.03a (ZT)1304, Br.03a (ZT)1305, Br.04a } \\
\text { (ZT)1311, Br.04a (ZT)1312, Br.04a (ZT)1315, }\end{array}$ \\
\hline 2 & Moderately resistant & $11-20$ & 11 & $\begin{array}{l}\text { Br.05a (ZT)1322, Br.05a (ZT)1325, Br.14a } \\
\text { (ZT)1334, Br.14a (ZT)1335, Br.14a (ZT)1336, } \\
\text { Br.14a (ZT)1338 }\end{array}$ \\
\hline 3 & Moderately susceptible & $21-40$ & 5 & $\begin{array}{l}\mathrm{Br} .14 \mathrm{a}(\mathrm{ZT}) 1331, \mathrm{Br} .14 \mathrm{a}(\mathrm{ZT}) 1337, \mathrm{Br} .14 \mathrm{a} \\
\text { (ZT)1339, Br.15a (ZT)1355, Br.15a (ZT)1356 }\end{array}$ \\
\hline 4 & Highly susceptible & $>40$ & 2 & Br.14a (ZT)1332, Br.14a (ZT)1333 \\
\hline
\end{tabular}

formation time, later decreased disease severity was noticed at maturity. The maximum mean per cent disease index of 41.17 was recorded in the entry $\mathrm{Br} .14 \mathrm{a}$ (ZT)1333 followed by mean per cent disease index of 40.06 in the entry Br.14a (ZT)1332. The least PDI of 0.58 was recorded in Br.03a (ZT)1303. Initially, higher disease incidence of $37.28 \%$ and $32.72 \%$ was recorded in $\mathrm{Br}$.14a (ZT)1333 and Br.14a (ZT)1332, respectively, at 45 days after sowing whereas only $<20.00 \%$ of disease reported in the remaining entries were moderately resistant.

The development of disease at early stage is less and starts increasing with increase of age because of susceptibility towards the disease. Several authors have reported different disease severity of Alternaria leaf spot during crop growth period of cotton. Zhu et al. (2019) reported incidence of $100 \%$ leaf blight symptoms at late growth stage of cotton. The increased disease severity at maturity period could be due to availability of suitable micro-climate for the disease occurrence and susceptibility of the lines for the disease. The prevailed micro climate within the lower canopy is more favorable for disease development and higher green boll loads are more susceptible to disease (Harde et al., 2016). The favorable environmental condition plays a vital role in occurrence of leaf blight disease in cotton and their incidence increases year by year due to susceptibility of cultivars. Bhuiyan et al. (2007) reported that species of $A$. macrospora was most prevalent during early growth period and $A$. alternata species was more prevalent during the end of growing season responsible for defoliation and higher disease severity. Le and Gregson (2019) reported that, severe disease outbreaks were recorded in multiple cotton crops in south Wales (NSW) in the 2017/18 season.

The Manifestation of plant pathogens is most important cause of losses in several crop. The occurrence of foliar disease reduces the production and productivity in cotton (Bernardes et al., 2013). In this study, the least seed cotton yield of $143.67 \mathrm{~g}$ per plant was obtained in $\mathrm{Br}$.14a (ZT)1333 and higher seed cotton yield of $208.67 \mathrm{~g}$ per plant was reported in Br.03a (ZT) 1308 (Table1). The occurrence of disease in $\mathrm{Br}$.14a (ZT)1333 reported maximum as compared to other breeding entries. The higher severity of disease was reported due to defoliation and reduced photosynthetic activities leads to reduced yield in cotton. Infection due to pathogenic Alternaria results in pre-mature defoliation in cotton (Rajesha et al., 2012) and causes quick and severe defoliation responsible for yield losses (Zhao et al., 2013). Saravanan et al. (2015) reported that incidence of leaf blight caused severe yield loss in cotton during the year of 2013-2014. The correlation between disease development and yield is most often summarized as a simple empirical model that describes average performances of the crop during the presence of virulent pathogen. Understanding the influence of weather factors on host stage and disease development is prerequisite to strategically manage these diseases (Bhattiprolu and Monga, 2018). In this study, correlation coefficient was determined between the yield and leaf blight disease severity among all the entries (Table 1).

The result revealed that the level of disease severity was negatively correlated to yield $r=-0.86$ ). It was summarized that every one per cent increase of disease incidence leads to reduction of $0.86 \mathrm{~g}$ of seed cotton yield. The higher incidence of disease recorded the least cotton yield in $\mathrm{Br}$. 14a (ZT) 1333. The severity of disease and yield loss reported by Bhattiprolu (2018) revealed that the occurrence of $35 \%$ of Alternaria leaf blight disease was responsible for yield loss of $13.80 \%$. Chattannavar et al. (2009a) reported that economic yield loss of $26 \%$ was reported due to Alternaria leaf blight in cotton. Higher severity of disease is directly correlated to yield loss in cotton. Bhattiprolu and Monga 
(2018) reported that significant Alternaria disease correlation of $r=0.984$ was developed due to prevalence of weather factors in kharif 2017. In both 2016 and 2017, high rainfall than normal occurred in New Mexico in the fall, which caused high levels of Alternaria leaf blight incidence leads to severe foliar damage and yield loss (Zhu et al., 2018). Utilization of developed resistant cultivars in farming is the simplest, effective and inexpensive method of disease management.

In addition to this, use of disease resistant cultivars conserves resources, other inputs and effectively minimizes the production cost, time and sources of energy when compared to other management practices of disease. In this study, thirty-nine genotypes were categorized into different groups based on disease reaction (Table 2). Among them, none of the genotypes showed apparently immune reaction to Alternaria leaf blight in cotton. However, twenty-one genotypes showed resistant reaction to leaf blight infection and eleven genotypes viz., $\mathrm{Br}$.03a (ZT)1304, Br.03a (ZT)1305, Br.04a (ZT) 1311, Br.04a (ZT)1312, Br.04a (ZT)1315, Br.05a (ZT)1322, Br.05a (ZT)1325, Br.14a (ZT)1334, Br.14a (ZT)1335, Br.14a (ZT)1336, Br.14a (ZT)1338 showed moderately resistant reaction. Further, five genotypes (Br.14a (ZT)1331, Br.14a (ZT)1337, Br.14a (ZT)1339, Br.15a (ZT)1355, Br.15a (ZT)1356) showed moderately susceptible reaction, however, two genotypes $\mathrm{Br}$.14a (ZT)1332 and $\mathrm{Br}$.14a (ZT)1333 showed of highly susceptible reaction to leaf blight. However, screening of cotton against Alternaria resistance can be easily incorporated into breeding program is an essential component, as was the case in this study.

In this study and also previous studies (Hosagoudar 2007; Anil 2013; Cia et al., 2016), none of the cotton genotypes were identified as immune to Alternaria leaf blight whereas several Pima cotton cultivars were evaluated for resistance to Alternaria leaf blight, based on disease severity rating, lesion size and numbers and Pima was found to be more susceptible (Zhu et al., 2018). Similarly, Chattannavar et al. (2009b) screened 196 cotton cultivars under field conditions for resistance to Alternaria blight during the kharif season of June 2007. Among them, nine cultivars viz., DCH 32, RAMSHH 7, GSHB 895, CCHB 2628, CCCHB 07-2, DHB 0782, NSPL 414, Ajeet 999 and HAGHB 12 were found to be most resistant to disease. Zhu et al. (2018) evaluated 133 cotton genotypes for Alternaria leaf spot resistance and found that none cotton genotypes were immune to disease caused by A. alternata. Sandipan et al. (2017) reported that 21 of 38 entries were disease free from apparent Alternaria leaf blight in natural condition in India.

However, infection of Alternaria spp. (A. macrospora and $A$. alternata) is known to cotton and expression of typical symptom may be absent. Furthermore, the disease free conditions in some genotypes may be due to disease escape mechanism or field resistance, which is especially true when disease is not prevalent. Even-though, our study shows that breeding entries were resistant and moderately resistant to disease; and resistant entries may be good source of resistance in breeding programmes. The study revealed that 21 breeding entries were identified as resistant to leaf blight disease. The breeding lines Br.14a (ZT)1333 and Br.14a (ZT)1332 were highly susceptible to leaf blight disease. The present study clearly indicates that in spite of remarkable efforts required for breeding for disease resistance, availability of resistant genotypes to Alternaria leaf blight is more advantageous in event of epidemics of leaf blight of cotton.

\section{Acknowledgments}

The authors are thankful to the Department of Cotton, TNAU, Coimbatore, India for providing facilities to carry out field research work. Authors are also grateful to the Indian Council of Agricultural Research (ICAR), New Delhi for the research grant as Senior Research Fellowship.

\section{Add-on Information}

Authors' contribution: G. Rajesha: Methodology, investigation, Formal analysis, Writing-original draft preparation, Funding acquisition, S. Nakkeeran: Conceptualization and supervision, Funding acquisition, Resources, T. Indumathi: Methodology, investigation, Formal analysis, P. Adhipathi: : Methodology, Writing - original draft preparation, A. Chandrasekar: Resources, Conceptualization supervision.

Research content: The research content of manuscript is original and has not been published elsewhere.

Ethical approval: Not applicable

Conflict of interest: The authors declare that they have no conflict of interest.

Data from other sources: Not applicable

Consent to publish: All authors agree to publish the paper in Journal of Environmental Biology.

\section{References}

Bashi, E., J. Rotem, H. Pinnschmidt and J. Kranz: Influence of controlled environment and age on development of Alternaria macrospora and on shedding of leaves in cotton. Phytopathology, 73, 11451147 (1983).

Bernardes, A.A., J.G.Rogeri, R.B. Oliveira, N. Marranghello and A.S. Pereira: Identification of foliar diseases in cotton crop. In: Topics in Medical Image Processing and Computational Vision. pp. 67-85 (2013).

Bhattiprolu, S.L. and M.P.P. Rao: Evaluation of cotton genotypes against Alternaria and Helminthosporium leaf spot diseases. J. Cott. Res. Develop., 27, 302-304 (2013).

Bhattiprolu, S.L. and D. Monga: Effect of weather parameters on the development of Alternaria leaf spot and grey mildew in cotton. $J$. Agrometeorol., 20, 315-318(2018).

Bhattiprolu, S.L. and M.P.P. Rao: Estimation of crop losses due to Alternaria leaf spot in cotton. J. Indian Soc. Cotton Impr., 14, 151154 (2009). 
Bhattiprolu, S.L.: Evaluation of Pyraclostrobin 20\% WG for the control of fungal diseases in cotton. Int. J. Chem. Studi., 6, 607-610 (2018).

Bhuiyan, A.S., M.C. Boyd, C. Martin and M. Hearnden: Development of Alternaria leaf blight on north Australian cotton (Gossypium hirsutum), species prevalence, and its control using mancozeb. Aus. Pl. Pathol., 36, 488-497 (2007).

Bodhke, V.S., C.U. Patil and S.B. Zade: Screening of different genotype of cotton for resistance against Alternaria leaf blight disease. Int. J. Chemi. Studi., 7, 1426-1428 (2019).

Chattannavar, S.N., G.N. Hosagoudar, R. Ammajamma and S.A. Ashtaputre: Survey for diseases of Bt cotton in north Karnataka. J. Cotton Res. Dev., 23, 156-158 (2009a).

Chattannavar, S.N., G.N. Hosagoudar, S.A. Ashtaputre and R. Ammajamma: Evaluation of cotton genotypes for grey mildew and Alternaria blight diseases. J. Cotton Res. Dev., 23, 159-162 (2009b).

Cia, E., M.G. Fuzatto, J.I. Kondo, L.H. Carvalho, M.F. Ito, F.L.F. Dias and P.B. Gallo: Response of cotton genotypes to theincidence of Alternaria leaf spot. Summa Phytopathol., 42, 357-359 (2016).

Consortium, R.E.X.: Combining selective pressures to enhance the durability of disease resistance genes. Front. Plant Sci., 7, 1916 (2016) doi: 10.3389/fpls.2016.01916

Cui, Y., A.A. Bell, O. Joost and C. Magill: Expression of potential defense response genes in cotton. Physiol. Mol. Pl. Pathol., 56, 25-31 (2000).

Fitt, G., C. Mares and G. Constable: Enhancing host plant resistance of Australian cotton varieties. Austra. Cotton Grow., 23, 20-26 (2002).

Gomez, K.A. and A.A. Gomez: Statistical Procedure for Agricultural Research. John Wiley and Sons, New York. (1984).

Gulhane, V.A. and A.A. Gurjar: Detection of diseases on cotton leaves and its possible diagnosis. Int. J. Image Proc., 5, 590-598 (2011).

Harde, S.N., L.V. Shinde and R.J. Chavan: Disease scenario on Bt cotton from Jalna district (MS), India. Proceeding of National Conference on Environment and Development, Lonavala, pp. 57-63 (2016).

Hillocks, R.J.: Alternaria leaf spot of cotton with special reference to Zimbabwe. Tropical Pest Managem., 37, 124-128 (1991).

Kaur, M. and N.K. Aggarwal: First report of Alternaria spp. causing leaf blight disease on Parthenium weed in India. New Dis. Rep., 31, 25 (2015)

Le, D.P. and A. Gregson: Alternaria leaf spot of cotton seedlings grown in New South Wales, Australia is predominantly associated with Alternaria alternata. Australian Plant Pathol., 48, 209-216 (2019).

Leff, B., N. Ramankutty and J.A. Foley: Geographic distribution of major crops across the world. Global Biogeoche. Cycles, 18, 1-27 (2004). doi:10.1029/2003GB002108
Newman, M.A.: Cotton disease and nematode control. Extn. PI. Path. Info., 24, 1-11 (2011).

Padaganur, G.M., R.V. Hiremath and M.K. Basavaraj: Estimation of yield loss due to Alternaria leaf spot and blight in cotton. J. Cotton Res. Dev., 14, 144 (1989).

Rajesha, G., S. Nakkeeran and A. Chandrasekaran: Genetic diversity of Alternaria isolates causing leaf blight of cotton and management of leaf blight with bacterial endophytes. Natio. Acad. Biol. Sci. Newsletter, 3, 6 (2012).

Rajesha, G., S. Nakkeeran, T. Indumathi, P. Adhipathi and A. Chandrasekar: Evaluation of different fungicides against Alternaria macrospora Zimm. inciting leaf blight in cotton under in vitro. Int. J. Curr. Microbiol. App. Sci., 9, 1051-1058 (2020).

Rotem, J., J. Eidt, U. Wendt and J. Kranz: Relative effects of Alternaria alternata and $A$. macrospora on cotton crops in Israel. Plant Pathol., 37, 16-20 (1988).

Sandipan, P.B., G.R. Bhanderi, R.D. Patel, D.M. Patel and B.G. Solanki: Screening of varieties/ breeding materials for resistance to different diseases in natural condition under south Gujarat region. Int. J. Curr. Microbiol. App. Sci., 6, 1355-1361 (2017).

Saravanan, P., S. Divya, P. Venkatesan, R. Tanwar, A. Birah, C. Chattopadhyay, P. Jeyakumar and A. Barik: Trend analysis of pests and diseases complex in Bt cotton. J. Crop Prot., 4, 431-439 (2015).

Sheo, R.: Grading for cotton disease, Nagpur, CICR, Tech Bull., pp. 1-7 (1988).

Singh, K. and P. Rathore: Planting geometry and nutrient levels affecting seed cotton yield, productivity indices and economic parameters of Bt cotton (Gossypium hirsutum L.). J. Environ. Biol., 41, 1550$1559(2020)$

Vander, J.D.L., L.M. Deck and R.E. Royer: Gossypol prototype of inhibitors targeted to dinucleotide folds. Curr. Medicinal Chem., 7, 478-498 (2000).

Wheeler, T.A., J.R. Gannaway and K. Keatin: Identification of resistance to Thielaviopsis basicola in diploid cotton. Plant Dis., 83, 831-833 (1999).

Zhao, J., F. Zhao, G. Jian, Y. Ye, W. Zhang, J. Li and F. Qi: Intensified Alternaria spot disease under potassium deficiency conditions results in acceleration of cotton (Gossypium hirsutum L.) leaf senescence. Aust. J. Crop Sci., 7, 241(2013).

Zhu, Y., P. Lujan, S. Dura, R. Steiner, J. Zhang and S. Sanogo: Etiology of Alternaria leaf spot of cotton in southern New Mexico. Plant Dis., 103, 1595-1604 (2019)

Zhu, Y., P. Lujan, S. Dura, R. Steiner, T. Wedegaertner, J. Zhang and S. Sanogo: Evaluation of commercial upland (Gossypium hirsutum) and Pima ( $G$. barbadense) cotton cultivars, advanced breeding lines and glandless cotton for resistance to Alternaria leaf spot (Alternaria alternata) under field conditions. Euphytica, 214, 147(2018). 